\title{
La fiscalidad y el género: una relación tan necesaria como perentoria
}

Taxation and gender: a relationship as necessary as peremptory

\author{
Maite Vilalta Ferrer \\ Vicerrectora de Igualdad y Acción Social \\ Secretaria General de la Universidad de Barcelona \\ Profesora Titular de Economía aplicada \\ Universidad de Barcelona \\ E-mail: $\underline{\text { mvilalta@ub.edu }}$

\section{Pilar Rivas Vallejo} \\ Catedrática de Derecho del Trabajo y de la Seguridad social. \\ Delegada del Rector para la dirección de la Unidad del Igualdad \\ Universidad de Barcelona \\ E-mail: pilar.rivas.vallejo@ub.edu
}

Hablar de fiscalidad nos conduce a hablar de la existencia de gobiernos, de sector público. Es obvio que no existirían tributos si no existiera el sector público, de manera que la primera pregunta que debemos formularnos es por qué debe existir el sector público y cuál es su papel. Según el cuerpo teórico de la Economía Pública, este debe existir para desempeñar, fundamentalmente, tres funciones:

a) La primera función consiste en corregir los fallos del mercado a la hora de asignar eficientemente los factores de producción y los bienes y servicios de una economía. 
El mecanismo de mercado falla en demasiadas ocasiones, o, dicho de otro modo, solo proporciona asignaciones eficientes cuando se dan unos supuestos muy restrictivos, los de la competencia perfecta. No funciona cuando se trata de asignar bienes públicos, o cuando existen externalidades negativas o positivas en la producción y/o consumo de determinados bienes, o cuando la información que tienen los demandantes y productores acerca del bien o factor que se intercambian es imperfecta y/o asimétrica, o cuando el mercado de un bien o un factor es incompleto, es decir, cuando no están todos los posibles oferentes o demandantes del mismo, etc. El listado de fallos es aún más extenso y, en todos los casos, se reclama la intervención del sector público, pidiéndole que los corrija, que consiga asignaciones eficientes desde un punto de vista colectivo.

Las ineficiencias que se producen en el mercado laboral serían tan solo un ejemplo de la necesaria intervención del sector público en este ámbito, dado que algunas de las cuestiones que se plantean son: ¿por qué el mercado asigna salarios distintos a los hombres y a las mujeres? ¿Cuál sería el incremento de bienestar colectivo que se produciría si hombres y mujeres tuvieran igualdad de acceso al mercado laboral? Conseguir una asignación eficiente en el mercado laboral pasaría por asegurar, entre otros aspectos, que se trata de un mercado completo en el que participan todos/as los/as demandantes y oferentes de trabajo, y en el que el salario fuera aquel que internalizara los beneficios que supone que tanto hombres como mujeres pudieran participar en él con las mismas condiciones. Está claro que no habrá ninguna mano invisible que conduzca a este mercado a una situación como la descrita. Hará falta una acción gubernamental.

b) La segunda función que se atribuye al sector público es la de conseguir una distribución equitativa de la renta y la riqueza entre la ciudadanía. Se parte de la constatación de que el mecanismo de mercado proporciona una distribución de la renta y de la riqueza desigual, inequitativa. Y ello es así porque las rentas que obtenemos los ciudadanos a través del mercado dependen de nuestra dotación de factores de producción (trabajo y capital) y de la remuneración que obtenemos por ellos. Es decir, por un lado, la renta que un ciudadano puede obtener yendo al mercado laboral depende de la cantidad (dotación) de trabajo que pueda ofrecer y del salario por hora que obtenga por él y de los factores cualitativos que le aporte como valor añadido. Y, por otro lado, la retribución que pueda obtener a través de sus bienes inmuebles o activos financieros (su capital) dependerá también de la 
cantidad que disponga de ellos y de su rentabilidad. Y es obvio que todo ello no está distribuido siguiendo patrones o pautas de igualdad. Por ejemplo, la cantidad de trabajo que un individuo pueda ofrecer al mercado dependerá de varios factores, entre ellos, del género (entendido como «los papeles, comportamientos, actividades y atribuciones socialmente construidos que una sociedad concreta considera propios de mujeres o de hombres», Convenio del Consejo de Europa sobre prevención y lucha contra la violencia contra las mujeres y la violencia doméstica, artículo 3). Si la sociedad asigna a las mujeres las tareas relacionadas con el cuidado de nuestros mayores, de las personas dependientes y de los menores, su capacidad de ofrecer trabajo se verá mermada en relación a la de los hombres, es decir, su dotación de factor trabajo será menor. Y no solo esto, sino que, probablemente, lo acabará ofreciendo a sectores productivos de la economía donde los salarios son más bajos y las condiciones laborales más precarias.

La función del sector público, en este caso, es la de conseguir modificar la distribución inicial de la renta y la riqueza (la que proporciona el mercado), haciéndola más igual, más equitativa. O conseguir que la distribución inicial ya sea más igual ab initio.

c) Y la tercera función atribuida al sector público consiste en estabilizar el crecimiento económico, es decir, contribuir a que el crecimiento de la economía sea equilibrado, sostenido y sostenible. En este caso, se le pide al sector público que actúe evitando, por un lado, los comportamientos cíclicos de la economía y, por otro lado, aquellos comportamientos no respetuosos con el medio ambiente. Se trata, en definitiva, de no crecer a cualquier precio.

El crecimiento económico no es equilibrado si se consigue, por ejemplo, a costa de destruir puestos de trabajo, es decir, si aboca a la ciudadanía al desempleo. Y en este sentido, también el papel del sector público puede tener efectos en términos de género: ¿qué tipo de puestos de trabajo son los más vulnerables ante una posible recesión o crisis económica? ¿son los ocupados mayoritariamente por mujeres? Está claro que las políticas ocupacionales que se puedan llevar a cabo para conseguir estabilizar la económica no son neutrales en términos de género.

Estas son, descritas muy brevemente, las principales funciones asignadas al sector público. Y para que pueda llevarlas a cabo dispone de varios instrumentos, entre los cuales se encuentran los tributos, las políticas regulatorias, las ayudas públicas, las 
transferencias monetarias a ciertos colectivos de la población y a determinados sectores productivos, la provisión y/o producción de determinados bienes y servicios como son la educación, la sanidad, los servicios sociales, etc.

En definitiva, la cuestión es la siguiente: los gobiernos gastan para cumplir con las funciones que les han sido encomendadas, y para cubrir los gastos, disponen de unas fuentes de ingresos procedentes del poder tributario, regulatorio y expropiatorio que la ciudadanía, a través de procesos de elección democrática, les hemos otorgado. Los gobiernos tienen, pues, una doble actividad, la de gastar y la de ingresar. Y depende de cómo lleven a cabo una y otra, contribuirán con mayor o menor medida a la eficiencia asignativa (corrección de fallos del mercado), a la redistribución de la renta y la riqueza, y a la estabilización de la economía. Cuando hablamos de fiscalidad, nos referimos a ambas actividades, la de ingresar y la de gastar. Y ni una ni otra son neutrales en términos de género. Cualquier decisión que se tome puede tener efectos para la consecución de la plena igualdad entre hombres y mujeres.

El sector público cuenta con poderosos instrumentos para lograr cambiar las cosas y, en particular, para contribuir a alcanzar la igualdad entre hombres y mujeres. Según cual sea su política tributaria (su actividad de ingresar) y la finalidad de sus gastos (su actividad de gastar), se podrán conseguir asignaciones más o menos eficientes desde un punto de vista social (un mayor o menor bienestar colectivo), una distribución de la renta y la riqueza más o menos equitativa entre la ciudadanía (por ejemplo, entre hombres y mujeres), así como un crecimiento de la economía más o menos estable y respetuoso con el medio ambiente.

Depende de cómo estén diseñados los impuestos, estos acabarán siendo soportados en mayor o menor medida por una u otra parte de la ciudadanía. Sabemos que los impuestos, unos más que otros, se trasladan, es decir, no siempre son soportados, al cien por cien, por el sujeto pasivo legalmente obligado a pagarlos. El análisis de la incidencia impositiva permite arrojar luz sobre este asunto. Sabemos, por ejemplo, que los impuestos indirectos (como el IVA) son más trasladables que los directos (como el IRPF); sabemos que un impuesto que grave un bien o servicio cuya demanda es más inelástica que su oferta será relativamente más soportado por los demandantes del mismo (los consumidores) que por sus oferentes (los productores); sabemos que para diseñar un tributo progresivo debemos conseguir que la carga impositiva aumente más que proporcionalmente al aumento de la renta y/o la riqueza y que hay varios elementos 
de los impuestos que determinan este grado de progresividad (la tarifa impositiva, el mínimo exento, las desgravaciones o bonificaciones que se permitan aplicar, etc.); y sabemos que los tributos no son neutrales, sino que provocan cambios en las decisiones de los individuos; etc.

Los postulados de la incidencia impositiva resultan, pues, de gran utilidad para analizar el impacto de los tributos en términos de género, puesto que ayudan a dar respuesta a preguntas como: ¿quién acaba soportando más impuestos, los hombres o las mujeres? ¿Qué trato recibimos unos y otros en impuestos como nuestro IRPF? ¿A quiénes benefician relativamente más las desgravaciones y las bonificaciones establecidas? ¿Y en el IVA, quiénes acabamos consumiendo relativamente más aquellos bienes y servicios cuya demanda es más inelástica (más rígida) y, por tanto, estamos soportado relativamente más el impuesto? ¿Se observan conductas distintas, entre hombres mujeres, en relación al fraude y a la evasión de impuestos? Y si es así, ¿cuáles pueden ser los factores determinantes de dicho comportamiento?, etc. En esta línea, el informe de 2015 de ONU Mujeres titulado «El progreso de las mujeres en el mundo 2015-2016: transformar las economías para realizar los derechos» analiza cómo sería la economía si realmente fuese efectiva para las mujeres, creando sociedades más justas, y generando asimismo nuevos sectores de empleo (así, en la economía relacionada con los cuidados).

Este tipo de preguntas también deben realizarse desde la perspectiva del gasto público. Es decir, cabe preguntarnos cuál es el impacto de las políticas de gasto llevadas a cabo por los gobiernos: ¿a quién van destinadas? ¿Favorecen relativamente más a los colectivos más desfavorecidos? ¿Son las mujeres o los hombres los principales beneficiaros de dichas políticas? ¿Qué tipo de políticas públicas suponen incentivos que contribuyen a eliminar la brecha de género?, etc.

Tanto la actividad de gastar como la de ingresar de los gobiernos debe quedar debidamente reflejada en su presupuesto, pues cualquier decisión de un gobierno quedan reflejadas en dicho documento presupuestario. Y es por ello que hablamos de la necesidad de elaborar presupuestos públicos con perspectiva de género. Es necesario destacar qué partidas de gasto van directa o indirectamente dirigidas a disminuir la brecha de género, y qué políticas tributarias han sido diseñadas pensando también en la existencia de dicha brecha. Es necesario trabajar en el diseño de indicadores que acompañen y complementen cualquier documento presupuestario que sea aprobado por algún ente gubernamental, que permitan calibrar el impacto de las políticas públicas a 
corto, medio y largo plazo, de forma complementaria al necesario informe de impacto de género que prescribe la Ley 30/2003, de 13 de octubre, para cualquier instrumento normativo.

En este sentido cabe destacar que, desde 2008, se ha incorporado un Informe de Impacto de Género al Proyecto de Presupuestos Generales del Estado, como manda la Resolución del Parlamento Europeo, de 15 de enero de 2019, sobre igualdad de género y políticas fiscales en la Unión, a tenor del cual han de introducirse medidas de fiscalidad directa (impuesto sobre la renta de las personas físicas, impuesto de sociedades e imposición del capital y del patrimonio) y relativas a impuestos indirectos, así como considerar el impacto de la evasión y la elusión fiscales en la igualdad de género (una de las principales causas de desigualdad de género tanto en la UE como en el mundo, al disminuir los recursos públicos disponibles para actuar contra la desigualdad, como también se deriva de la falta de ajuste de los convenios de doble imposición), y, finalmente, también en las políticas fiscales, en cuyo ámbito se pide a la Comisión que promueva la igualdad de género. Sin duda este instrumento legislativo puede tomarse como línea hermenéutica para el despliegue de las acciones que puedan corresponder en los estados miembros en coherencia con la política de la UE. A título de ejemplo, el Parlamento Europeo, con especial referencia a la brecha salarial y de empleo entre hombres y mujeres, sobre la que bascula la política impositiva sobre la renta, subraya en su resolución que "los sistemas fiscales deben dejar de basarse en la asunción de que los hogares agrupan y comparten sus recursos por igual, y que la imposición individual es decisiva para lograr la justicia fiscal para las mujeres" e insta "a todos los Estados miembros a introducir progresivamente la imposición individual, garantizando la conservación plena de todas las prestaciones económicas y de otro tipo ligadas a la parentalidad en los sistemas fiscales actuales". De igual modo, pide que el impuesto sobre la renta de las personas físicas se conciba para actuar sobre dicha brecha y para subvertir los roles de género, que se evalúe el potencial impacto de los incentivos o desgravaciones fiscales a las empresas sobre la igualdad de género, o que se aplique eficazmente la Directiva 2004/113/CE del Consejo, de 13 de diciembre de 2004, por la que se aplica el principio de igualdad de trato entre hombres y mujeres al acceso a bienes y servicios y su suministro, especialmente en materia de seguros. En definitiva, constata el sesgo de género en las relaciones entre legislación fiscal y género, sus normas y su comportamiento económico y, en particular, “observa que el IVA ejerce un sesgo de género debido a las pautas de consumo de las mujeres, que difieren de las de 
los hombres en tanto que adquieren más bienes y servicios con el objetivo de favorecer la salud, la educación y la nutrición", lo que, unido a los ingresos inferiores de las mujeres, lleva a que estas soporten una mayor carga del IVA, por lo que también la política fiscal sobre el IVA puede ser un instrumento de acción positiva sobre las relaciones de desigualdad entre mujeres y hombres (en particular, insta a los estados miembros a la concesión de exenciones del IVA, tipos reducidos y tipo cero para los productos y servicios con efecto positivo para la sociedad, la salud o el medio ambiente, o, en concreto, la aplicación de un tipo $0 \%$ para los productos higiénicos que mitigue la pobreza menstrual, por no considerarse en algunos estados productos básicos, como todos aquellos relativos al cuidado de menores o de mayores).

Por otra parte, el Parlamento europeo destaca en la resolución citada el negativo impacto que la falta de transparencia de los datos y su desglose por géneros en materia de asignaciones y distribuciones de recursos del sistema fiscal provoca sobre la necesaria actuación en este campo, por lo que insta a los Estados miembros a que "recopilen datos fiscales sobre una base individual y no solo basándose en el hogar, y que reduzcan las brechas de género en cuanto a la información sobre patrones de consumo y el uso de tipos reducidos, sobre la distribución de las rentas empresariales y los pagos fiscales relacionados y sobre la distribución del patrimonio neto, los rendimientos del capital y los pagos fiscales relacionados".

En coherencia con este llamamiento europeo, recientemente, el enfoque de género figura de forma destacada y novedosa en las normas dictadas por el Ministerio de $\underline{\text { Hacienda español para la elaboración de los Presupuestos Generales del Estado para }}$ 2020, que fueron publicadas en el BOE (Orden HAC/20/2020, de 20 de enero). Con ello, se pretende dar un paso más e introducir de manera integrada la perspectiva de género en los programas presupuestarios y, por ende, en las políticas públicas de gasto. Para la elaboración de dicho informe se prevé la constitución de un grupo de trabajo integrado por representantes del Ministerio de Igualdad, de la Secretaría de Estado de Presupuestos y Gastos, de la Dirección General de Presupuestos y de la Dirección General de Costes de Personal.

La idea es, según se recoge en el informe final de 2005 del Grupo de especialistas del Consejo de Europa en presupuestación con perspectiva de género, que se realice una «evaluación de los presupuestos basada en el género, con la incorporación de una perspectiva de género a todos los niveles del proceso presupuestario y la 
reestructuración de los ingresos y los gastos con el fin de promover la igualdad de género» (definición de "presupuestación con perspectiva de género").

En el ámbito autonómico, tal como señalan De Villota, Jubeto y Ferrari (2009), el gobierno pionero en introducir el estudio del impacto de género de sus presupuestos fue el de la Comunidad Autónoma del País Vasco a través de una experiencia, llevada a cabo entre 1999 y 2002, que representó una prueba piloto imitada posteriormente por otros gobiernos. Lo siguió, por ejemplo, el gobierno de Andalucía, que, desde el ejercicio presupuestario de 2005, elabora un Informe de Evaluación de Impacto de Género que acompaña a la Ley de Presupuestos de dicha Comunidad Autónoma y que, desde el ejercicio 2009, en virtud de la Ley 3/2008, tiene rango de documentación anexa al anteproyecto de Ley del Presupuesto y, por tanto, se remite al Consejo de Gobierno para su aprobación. Más recientemente, en la Comunidad Valenciana también se elabora, desde el año 2014 (para el presupuesto de 2015), un Informe de Impacto de Género que acompaña el proyecto de presupuestos de la Generalitat Valenciana, en cumplimiento de lo establecido por la Ley 6/2013, de 26 de diciembre, de Presupuestos de la Generalitat.

En el ámbito local existen también, en esta línea de actuación, experiencias recientes como "El Informe de Evaluación del Impacto de Género del proyecto de Ordenanzas fiscales para el año 2020", elaborado por el Departamento de Transversalidad de Género del Ayuntamiento de Barcelona. También es el caso de los ayuntamientos de Madrid y de San Sebastián (Rodríguez Modroño, y Domínguez Serrano, 2009).

Solo avanzando en esta dirección se podrá comprobar qué efectos tiene la actuación del sector público en la disminución de las desigualdades entre hombres y mujeres. Únicamente así, conociendo y divulgando esta información de carácter presupuestario, la ciudadanía podrá pedir rendición de cuentas a sus gobiernos acerca de este gran reto que tiene planteado hoy la humanidad, y que ha quedado fijado como el objetivo número cinco (igualdad de género) de los llamados Objetivos de Desarrollo Sostenible acordados en 2015 por la ONU.

Los gobiernos, tanto supraestatales como estatales, regionales y locales, tienen una gran responsabilidad en la consecución de dicho objetivo, puesto que, tal como se ha descrito anteriormente, tienen en sus manos instrumentos fiscales de gran alcance para logarlo. Y cabe tener en cuenta que, cuando hablamos de sector público, usamos su acepción 
extensa, refiriéndonos a todo el conglomerado de administraciones y entidades que lo conforman. Y en este sentido, cabe recordar que las universidades públicas forman parte de este entramado, de manera que sería necesario que sus presupuestos fueran también elaborados con perspectiva de género, como algunas ya están haciendo o bien lo han incorporado como una de las actuaciones a desplegar en el marco de sus planes de igualdad. Por ello, algunas de nuestras universidades, dentro y fuera del país, han tomado la iniciativa en la elaboración de sus presupuestos en clave de género, así la Universidad de Granada (desde 2019), o la Universidad del País Vasco (desde 2011). Por su parte, otras, como la Universidad Pablo Olavide, o la Universidad de Barcelona, han comenzado a orientar sus presupuestos por esta senda en el año 2020. Sirvan las presentes páginas para efectuar la correspondiente llamada a dichas políticas, cuyo análisis excede del propósito de la presente breve presentación.

\section{Referencias}

De Villota, Paloma; Jubeto, Yolanda; Ferrari, Ignacio (2009), Estrategias para la integración de la perspectiva de género en los presupuestos públicos, Instituto de la Mujer (Ministerio de Igualdad).

Jubeto, Yolanda y Larrañaga, Mertxe (2011). Los presupuestos con enfoque de género en la UPV/EHU. Una primera aproximación, https://www.ehu.eus/documents/2007376/6552728/PEG-UPV-EHU-Primeraaproximacion.pdf.

Jubeto, Yolanda y Larrañaga, Mertxe (2016). Presupuestos con enfoque de género en la UPV/EHU Análisis del Capítulo I (Año 2013). Accesible en: https://www.ehu.eus/documents/2007376/6552728/PEGCapitulo-I-

\section{3.pdf/af7c4469-b269-42c1-bd94-060903648b45.}

Morales Caparrós, M. José, Esteban Pagola, Ana Isabel, y Luna Jiménez, María José (2010). "Diferencias entre metodologías para la evaluación del impacto de género en los presupuestos: su aplicación en la universidad". En Investigaciones multidisciplinares en género: II Congreso Universitario Nacional" Investigación y Género": Sevilla, 17 y 18 de junio de 2010 (pp. 733-754).

Rodríguez Modroño, Paula; Domínguez Serrano, Mónica (2020): Análisis de los presupuestos desde la perspectiva de género Universidad Pablo de Olavide. 
Vicerrectorado de Cultura y Compromiso Social, Oficina para la Igualdad, Universidad Pablo de Olavide, de Sevilla.

Rothe, A. et al. (2008). Gender budgeting as a management strategy for Gender Equality at universities: concluding project report. Munich: Frauenakad.

Steinpórsdóttir, F.S., Heijstra, T. M., Einarsdóttir, T y Péutrsdóttir G. (2016). Gender Budgeting in Academia-Toolkit. https://www.hi.is/sites/default/files/arnarg/genderbudgeting-in-academiatoolkit.pdf.

Steinpórsdóttir, F. S., Einarsdóttir, P., Heijstra, T. M., y Margrét Pétursdóttir, G. (2016). Money Talks: Gender Budgeting in the University of Iceland. Icelandic Review on Politics \& Administration, 12(1).

Universidad del País Vasco. Presupuestos con enfoque de género en la UPV/EHU (2011-2016). https://www.ehu.eus/es/web/berdintasunadireccionparalaigualdad/aurrekontuakgenero-ikuspegiarekin. Puede verse un análisis de los resultados de esta política en https://www.ehu.eus/es/web/berdintasuna-direccionparalaigualdad/aurrekontuakgenero-ikuspegiarekin y un informe de cada uno de los años desde 2011 en https://www.ehu.eus/es/web/berdintasuna-direccionparalaigualdad/dokumentuakpeg.

Universidad de Granada. Presupuesto para el ejercicio 2019 (Capítulo 6: Presupuesto con perspectiva de género en la Universidad de Granada), http://gerencia.ugr.es/pages/vger_eco/presupuestos/presupuesto2019definitivo/\%21 